\title{
A Proposal to Expand the Community of Users Able to Process Historical Rainfall Data by Means of the Today Available Open Source Libraries
}

\author{
Paolino Di Felice ${ }^{1}$, Luca Finocchio ${ }^{2}$, Daniele Leombruni ${ }^{2}$ and \\ Vittoriano Muttillo 2 \\ ${ }^{1}$ Department of Ingegneria Industriale e dell'Informazione ed Economia, University of L'Aquila, Italy \\ ${ }^{2}$ Master students in Engineering Informatics, University of L'Aquila, Italy
}

\begin{abstract}
The paper presents a software architecture based on open source technologies, implemented by the authors in an experience of processing spatio-temporal data gathered by rain gauges spread across two regions of central Italy. The interest in the automatic processing of data about precipitation is widespread, however, today only an inner circle of stakeholders can think of taking advantage of the available open source libraries because a strong programming skill is required to use them. It is opinion of the authors that the implemented software architecture is suitable for expanding the community of users able to process "by themselves" historical precipitation data. The centre of the architecture is the technology of the spatial database management systems. They offer full support for the creation and management of a spatial database suitable to store the rainfall data usually spread out in several files. Moreover, they allow adding to such a repository a large set of ad hoc "objects" oriented to carrying out spatio-temporal computations on the precipitation. The stakeholders are only required to familiarize with the database's objects and invoke their execution. A large part of the paper is devoted to show how the adopted conceptual setting can assist nontechnical users in carrying out personalized computations on rain gauges data. The computing needs posed by the experience described in this paper are common to many other areas of high social impact that involve spatio-temporal data, hence we believe that the implemented framework can be exported to them, keeping unaltered operational effectiveness.
\end{abstract}

Keywords: open source software, DBMS, SQL, PostGIS, R language, software architecture, user defined function, precipitation retrieval

\section{Introduction}

The wise management of water reserves, the prevention of natural disasters related to excessive rains, and the choise of the best land use in agriculture are few issues of primary importance to most countries. The modeling of these scenarios and the efficient management of the huge amount of spatio-temporal data involved are very complex problems that must be addressed with methods and tools from the areas of computing and information technology. Some of the recent contributions in this direction are: ( $\mathrm{Lu}$ and Wong, 2008; Krishnakumar et al., 2009; Li et al., 2011; Rowhania et al., 2011; Tabari and Talaee, 2011; Ahani et al., 2012; Bostan et al., 2012; Keblouti et al., 2012; Chen and Liu, 2012).

Unfortunately, in most of the IT approaches appeared so far the input data are dispersed in a multitude of independent files, sometimes conflicting even in their format. In this regard (Carleton et al, 2005) state the following. "Typically, hydrologic data are stored in a spreadsheet. To keep data organized in a spreadsheet, separate files are often created by the user to hold data from different locations and years. This results in an ever increasing number of files, each of which have to be individually managed whenever new data are added or new calculations desired. Problems also arise when attempting to compare data between two or more 
individual spreadsheet files. Spreadsheets also make it difficult to analyze data categorically, such as on a daily or monthly time step."

To overcome this limit, the software architecture we present in this paper, and implemented in a recent experience of storage, cleaning, processing and visualization of spatio-temporal data collected by rain gauges located on two regions of central Italy, puts at the centre the technology of the Spatial Database Management Systems (SDBMSs). This technology has dramatically increased the effectivness and efficiency in carrying out many everyday activties involving large structured datasets.

Our software architecture is based exclusively on open source technologies. Since several years ago there has been a consolidation of open source software able to manipulate spatiotemporal data. This fact, together with the appearance of international no-profit foundations (e.g., Open Source Geospatial Foundation - OSGeo), indicates that the interest in open source software is expected to grow significantly in the near future. Steiniger and Bocher (2009) recognize four indicators of this trend: (1) increasing number of projects run using open source GISs, (2) increasing financial support by government agencies, (3) increasing download rates, and (4) increasing number of use-cases. With regard to the geostatistical computations of rainfall data, in particular, the use of the R environment (Pebesma, 2004), extended with libraries such as gstat and raster, today appears as the best choice. Unfortunately, as stressed by Hengl (2009) - page 90, "the main problem with $R$ is that each step must be run via a command line, which means that the analyst must really be an R expert". This circumstance limits the use of this valuable software tool to subjects with strong programming aptitude.

Thanks to the possibility of formulating "powerful" spatial queries at a "high level" of abstraction, SDBMSs have the further merit of greatly extending the community of those who can interact with such a software technology to perform relevant spatial analysis without owning a specific IT skill. In the experience of computing of spatio-temporal data reported in this paper, the query expressiveness is obtained by placing side by side to the basic SQL operators and to the built-in functions of the SDBMS, "database objects" created ad hoc (technically they are User Defined Functions - UDFs), while the abstraction is obtained by masking to the end user the logical structure of the underlying database (for example, through the mechanism of the views), as well as the functions called in the query. From the stakeholders involved is only required to familiarize with the objects that the database exposes and invoke their execution. This level of competence can be achieved after a short on-the-job training of the nontechnical personal.

The paper is divided in two parts. The first part: a) proposes the technological solution setting and lists its several advantages over the current ways of processing historical data about precipitation (Section 2), b) describes the reference format of the rainfall data, the design of a Spatial DataBase (SDB) adequate to store the data about the geographical area of interest, the rain gauges spread over it, and the collected measurements (Section 3), c) gives details about the structuring of a number of packages that implement the computing requirements posed by the application domain we refer to (Section 4), and, finally, d) discusses the related work (Section 5). The second part of the paper (Section 6) shows, through a case study about rainfall data recorded by rain gauges located in central Italy, the simplicity with which, working from the inside the console of the SDBMS, one could take advantage of the many features offered by the packages we have developed (and displayed as database objects) to implement the phases ranging from the storage of the measurements in the $\mathrm{SDB}$, to their cleaning and processing, till the production of maps.

\section{The Solution Setting}

Figure 1 shows the reference software architecture of our solution setting. It can be interpreted at two different levels: one abstract, the other concrete. The abstract level highlights the basic elements of the solution: a SDBMS and a programming language oriented to geostatistics. Both elements are on top of a SDB containing the data about the geographic area of interest, the rain gauges located in it and the measurements collected. The concrete level, vice versa, details the software technologies chosen, i.e., PostgreSQL/PostGIS and PL/R (with the libraries gstat and raster). 
The central element of the architecture of Figure 1 is the technology of the SDBMSs. It offers enormous advantages over the current ways of processing the historical data about precipitation; in detail, it allows us:

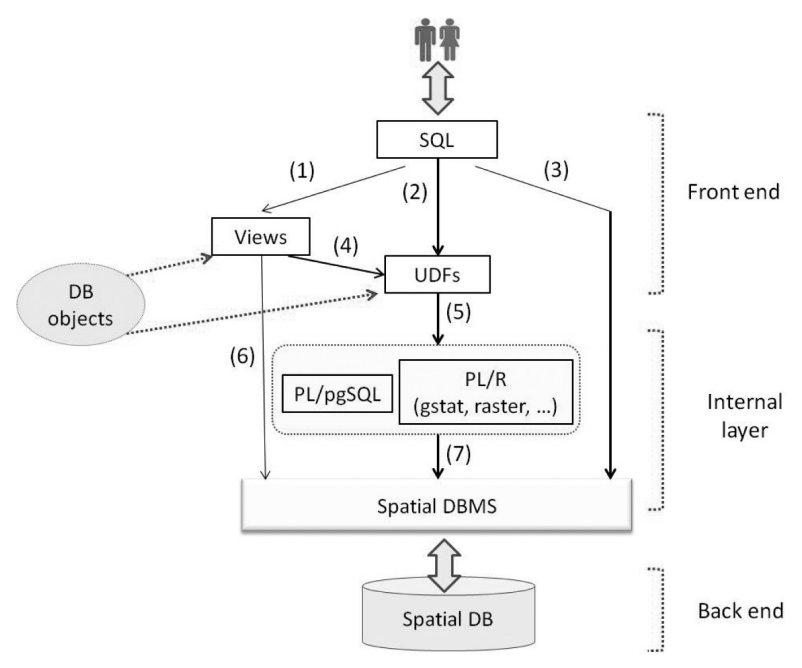

Figure 1. The reference software architecture.

1. to keep together, in a single container, all the historical measurements. Data typically dispersed in a multitude of independent files, sometimes conflicting even in their format;

2. to limit the access to the data only to people holding explicit granting privileges;

3. to call the spatial operators of the SQL language as well as the spatial built-in UDFs, when querying the SDB. UDFs are a relevant capability of SDBMSs. For example, PostgreSQL/PostGIS (ver. 9.2, PosGIS model: postgis_21_sample) offers 1092 built-in UDFs;

4. to code ad hoc views and UDFs (to be added to the built-in UDFs) to be exposed as database objects, both joinable in SQL queries of extraordinary expressiveness;

5. to transform the end users' rainfall data processing needs from programming activities, as it happens today, to querying activities against the SDB, with considerable masking of technicalities and, therefore, potentially widening the number of those who in the future could face such difficulties with profit. In fact, by taking advantage of the geostatistical and graphical functionalities of the $\mathrm{R}$ programming environment (and of the libraries linked to it, over all gstat and raster), with which the SDBMS communicates effectively, it is easy to greatly extend the capabilities of the SQL-pure UDFs (i.e., functions written by embedding SQL commands into the internal language of the SDBMS), greatly simplifying the job of the end users.

Figure 1 shows the paths through which users can interact with the SDB. In each path, it is required to write SQL queries to be executed in the console of the SDBMS. For users without any technical skill, the interaction with the rainfall data takes place by invoking the database objects (either path 1 or 2), while path (3) is for skilled users. Path (1) may involve objects of type view (path 1-6), or views and UDFs together (path 1-4-5-7), while path (2-5-7) involves only UDFs. If the user invokes in the query some UDFs, he/she, however, does not perceive their internal encoding, and even the coding language (either PL/pgSQL or PL/R).

To learn about the merits of the $\mathrm{R}$ environment, refer to (Hengl, 2009). The literature already proposes examples of usage of $\mathrm{R}$ to develop packages (e.g., Cannon, 2011).

The communication between PostgreSQL and $\mathrm{R}$ may take place either by keeping them separate, or by using $\mathrm{R}$ from inside PostgreSQL. Choosing the first option, they communicate by importing/exporting shapefiles: so, one can, for example, run a query in PostgreSQL, export the result as a shape, load it into $\mathrm{R}$ and, eventually run (in this environment) the processing he is interested in. But because, as already mentioned, work with $\mathrm{R}$ implies that the analyst must really be an $\mathrm{R}$ expert, we decided to adopt the second option in which the use of $\mathrm{R}$ is hidden to the end users of the SDB, therefore, they may not be programmers.

PostgreSQL allows us to interact with the $\mathrm{R}$ environment by using PL/R, i.e., a procedural language suitable for writing PostgreSQL functions in the programming language R. PL/R provides most of the R's functionalities.

\section{Format of the Precipitation Data and Structure of the Spatial Database}

In Italy, data about the precipitation over the territory are collected by different regional and national agencies without, however, their sharing either a standard methodology or a common 
data format. Burnette and Stahle (2013) report a similar situation for the United States. In this paper, we will refer to the data format adopted by the Italian Department of Civil Protection, which is a structure of the Presidency of the Council of the Ministers (http://www . protezionecivile.gov.it). Section 6.1 explains how our packages intercept the format heterogeneity in case the precipitation data come from a different source, allowing, therefore, to keep the structure of the SDB unaltered.

\subsection{Format of the Precipitation Data}

The rainfall data are collected in two categories of files. The first one consists of a single file about the stations of measures distributed over the land, while the second category is composed of many files as the months to which the measurements refer to. The rain gauges measure the precipitation volume (in $\mathrm{mm}$ ) each $15 \mathrm{~min}$ utes using a counter that is incremented by one each time it detects $0.1 \mathrm{~mm}$ of rain. The rain gauge calculates the precipitation accumulated in the last quarter of an hour by subtracting from the current value of the counter the value of the counter at the previous measurement, and dividing the result by 10 (to express the value in $\mathrm{mm}$ ).

The metadata about the rain gauges are described by the tuple: <id, name, station number, municipality, region, geographical position $>$ the latter expressed as latitude and longitude. The metadata about the rainfall measurements are described by the tuple: <rain gauge $i d$, data, hour, rainfall in 15 minutes $(\mathrm{mm})$, counter>.

\subsection{Structure of the SDB}

The SDB (called Rainfall_measurement) suitable to hold the data of interest is composed of the four tables of Figure 2. region and district refer, respectively, to the region and the municipality where the meteorological stations are located.

The SQL/DDL statements about the four tables of Figure 2 are given in the Appendix.

\section{Organization of the Packages}

The precipitation context raises many processing requirements. We discuss them in the following. The initial need consists of loading the measurements recorded by the rain gauges into the SDB. Subsequently, it is useful to perform an analysis about the distribution of the stations over the land. In fact, the sampling density plays a significant role in the performance of the spatial interpolation methods. Li and Heap (2008) report that "when data are sparse, the underlying assumptions about the variation among samples may differ and the choice of a spatial interpolation method and parameters may become critical."

The validation of the data about the precipitation is another crucial issue for increasing their quality. Data quality is among the major factors that affect the performance of the spatial interpolation methods (Li and Heap, 2008). Data noise can negatively affect the output of those methods.

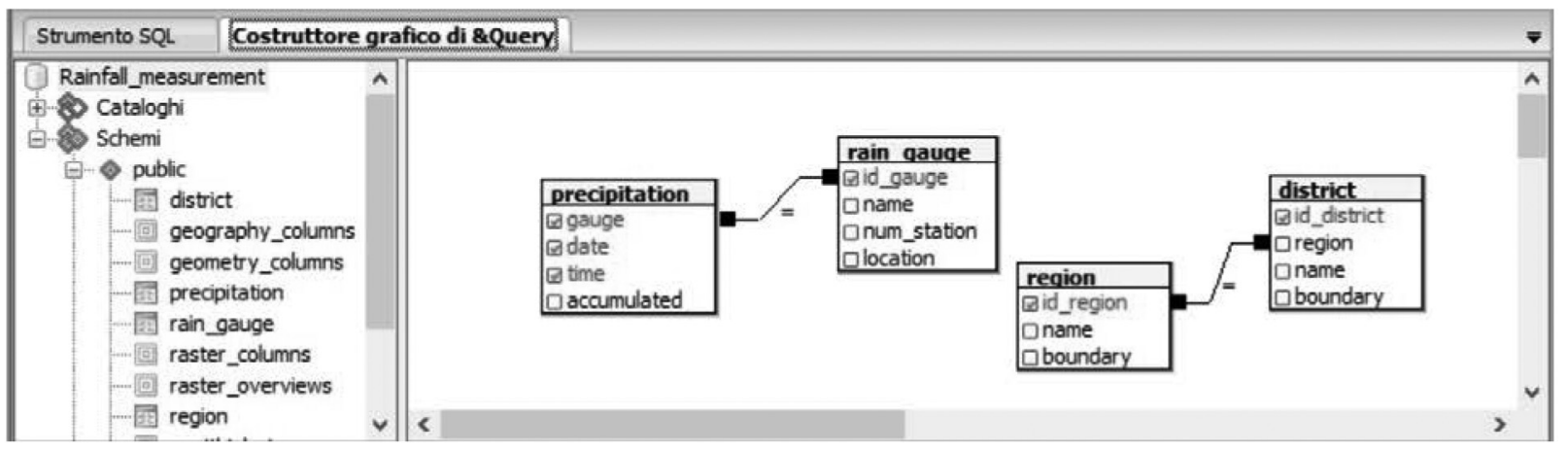

Figure 2. The four tables of the SDB. The red attributes are primary keys. The arcs connect the primary key to the corresponding foreign key. 
The construction of diagrams about the trend of the rainfall for time intervals selected on an hourly, daily, weekly, monthly or yearly basis is another crucial requirement.

Interpolation of the measured data over the geographical area with the ultimate goal of getting maps about the rainfall is fundamental. Interpolation techniques widely used are the inverse distance interpolation (IDW) method and the Kriging (Li and Heap, 2008). The cross validation of the interpolation carried out is mandatory to evaluate the results.

Table 1 brings together the processing requirements and the name of the corresponding package of PostgreSQL UDFs that implements them.

The Auxiliary package contains 34 functions created to support those contained in the other five packages. Table 2 lists the 34 functions divided according to the used coding language (namely: PL/R or PL/pgSQL). The names of these functions are left intentionally generic (UDF_0, UDF_1,...) so as not to create confusion or conflict with the name of the functions that invoke them.

Table 3 shows, for each package, the number of internal UDFs, the total number of lines of code, the number of UDFs of the package Auxiliary called. From Table 3 we learn about the massive use that the UDFs of the various packages make of the UDFs of the Auxiliary package.

The PL/pgSQL code refers to version 9.2 of PostgreSQL and to version 1.8 of PostGIS, while the PL/R code refers to version 8.3.0.14.

\begin{tabular}{|l||c|}
\hline The processing requirements & The package of UDFs \\
\hline \hline $\begin{array}{l}\text { Loading the precipitation data into the SDB } \\
\text { Cleaning of the precipitation data } \\
\text { Analysis of the distribution of the rain gauges over the Region }\end{array}$ & Basic \\
\hline $\begin{array}{l}\text { Construction diagrams about the rainfall for different time intervals } \\
\text { (hours, days, weeks, months, years) }\end{array}$ & Plotting \\
\hline Interpolation of the precipitation data over Region & IDW, Kriging \\
\hline Cross validation of the interpolation & CrossValidation \\
\hline & Auxiliary \\
\hline
\end{tabular}

Table 1. The processing requirements. Region denotes a generic geographic area.

\begin{tabular}{|c||c|}
\hline Language & Name of the UDF \\
\hline \hline PL/R & UDF_0, UDF_6, $\ldots$, UDF_14, $U D F \_16, \ldots$, UDF_23, UDF_27, .., UDF_31 \\
\hline PL/pgSQL & UDF_1 $\ldots$, UDF_5, UDF_15, UDF_24, UDF_25, UDF_26, UDF_32, UDF_33, UDF_34 \\
\hline
\end{tabular}

Table 2. List of the UDFs in the Auxiliary package.

\begin{tabular}{|c||c|c|c|}
\hline Package & Total \# of UDFs & Total \# of lines of code & $\begin{array}{c}\text { Total \# UDFs of the } \\
\text { package Auxiliary called }\end{array}$ \\
\hline \hline Basic & 7 & 205 & 1 \\
\hline Plotting & 20 & 604 & 36 \\
\hline IDW & 4 & 206 & $(4 * 4=) 16$ \\
\hline Kriging & 16 & 760 & $(16 * 4=) 64$ \\
\hline CrossValidation & 12 & 421 & $(12 * 2=) 24$ \\
\hline Auxiliary & 34 & 1026 & \\
\hline
\end{tabular}

Table 3. Numbers about the implemented packages. 


\section{Related Work}

(Ward et al., 1996), a pioneering work in the field of geocomputing, outline a computational architecture suitable to deal with the complexity and the diversity of data and processing needs in geoscientific data analysis. One of the key elements of the proposed architecture is the layer about the management of data. The architecture we implemented to carry out the experience about spatio-temporal raing gauges data intercepts this recommendation.

In (Shen et al., 2005), a relational database is introduced as the backbone of a system to deal with hydrological data." Authors attribute three major merits to their system's underlying database-supporting architecture: a) "great flexibility for user manipulation and customization"; b) "the great potentiality deriving from using the built-in database functions"; c) "the database builds a linkage among users' inputs, model data management, and the numerical model by implementing a set of dynamic database queries." The above three merits apply to our solution as well.

In the same year, (Carleton et al., 2005) developed a Microsoft Access database designed to facilitate the storage, retrieval and analysis of hydrologic data as an alternative to the prevalent trend at the time of storing such data in spreadsheets which, as already pointed out, suffer from severe limits. Besides presenting the database structure, (Carleton et al., 2005) provide a set of queries useful to retrieve data that involve calculations, comparisons and basic quality assurance/quality control calculations. Our experience reaffirms the relevance of querying, besides showing a way to deeply enhance its expressiveness by calling ad hoc UDFs and/or views.

Pokorny (2006) remarks that since the 1960s there is an ever increasing environmental demand from customers, authorities and governmental organizations. Then, he states that "A natural idea is that environmental information systems based on advanced database technologies could help to deal with these issues." Our practical experience builds on both the above statements.

\section{The Case Study}

This section shows the ease of use, as well as the effectiveness, of the UDFs collected in the six packages developed (Table 3), to support the archiving, cleaning and processing of precipitation data up to the production of rainfall maps. The discussion will involve only some of the UDFs.

The case study takes into account the measurements recorded in November 2007 by 137 meteorological stations, equipped with a rain gauge, located in the regions of Lazio and Umbria (central Italy). The dataset about the rain gauges and that about the measurements are in the .csv format and follow the pattern described in Section 3.

\subsection{SDB Loading}

Preliminarily, the . csv file about the rain gauges and the file about the measurements were both converted (by means of QGIS) into shape files. The shape files containing the geometry of the Italian regions and municipalities were downloaded from ISTAT (http://www.istat.it/). The format of the geometric data in all the shape files was expressed in the WGS 84 Spatial Reference System. Then, we imported (using the appropriate pgAdminIII plug-in) the content of the shape files about the rain gauges, regions and municipalities inside, respectively, tables rain_gauge, region and district of the SDB Rainfall_measurement. The manual actions mentioned so far are part of what we might call phase zero. From here on out, all the actions that will be described are greatly facilitated by the use of the UDFs developed.

To load the contents of the file of the measurements in the table precipitation is sufficient to invoke the UDF import_precipitation() of the package Basic (written in pure SQL Appendix) which, in turn, engages the UDF_0 (written in PL/R) of the Auxiliary package. UDF_0 also accounts for replacing with NULL missing and negative values. Any changes in the format of the file of the measurements, which in practice is frequent, entail changes to the body of UDF_0, but not to the structure of the SDB which, therefore, remains stable. The loading 
is obtained by running (in the query window of pgAdminIII) the SQL command:

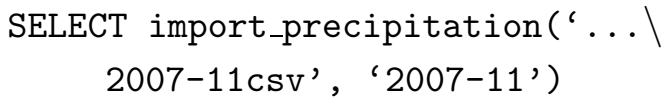

This command must be invoked as many times as the months of the measurements to be loaded into the SDB (Section 3).

The invocation of any one of our UDFs has to be made in the same operating mode. In the following we will avoid repeating it.

\subsection{Assessment of the Spatial Distribution of the Rainfall Stations Over the Territory}

Figure 3 shows that spatial distribution of the 137 meteorological stations is not uniform; in particular, in the case of the Lazio region, there is a high density of rainfall stations around Rome. In Lazio $\left(17,236 \mathrm{~km}^{2}\right)$ there are 84 stations, in Umbria $\left(8,456 \mathrm{~km}^{2}\right) 47$.

Each dot represents one station. Some stations are located slightly outside the two regions ( 3 in Tuscany, 2 in Abruzzo and 1 in Campania). A confirmation of what appears visually can be obtained by running the query SELECT count(*) FROM rainGauges_filter('LAZIO'), where rainGauges_filter() is a UDF of the package Basic.

As already mentioned (Section 4), the number of the meteorological stations and their distribution over the land impact the performance of the spatial interpolation methods ( $\mathrm{Li}$ and Heap, 2008). Table 4 shows that each rain gauge in Lazio covers, on the average, about $205 \mathrm{~km}^{2}$ while in Umbria it covers about $180 \mathrm{~km}^{2}$. These data confirm that the number of rain gauges in Umbria is more appropriate than that in Lazio. In both cases, the numbers confirm a trend well known in the literature, namely that frequently the number of stations is very low when compared to the size of the territory. For example, Bostan et al. (2012) report on a study about the annual precipitation in Turkey carried out by starting from the measurements acquired in just 225 meteorological stations throughout the entire country (an area of $783.562 \mathrm{~km}^{2}$ ). Therefore, it is legitimate to conclude that the situation in Lazio and Umbria is definitely more favorable.

\begin{tabular}{|c||c|}
\hline Region & Rain gauges distribution $\left(\mathrm{km}^{2}\right)$ \\
\hline \hline Lazio & 204.7 \\
\hline Umbria & 179.8 \\
\hline
\end{tabular}

Table 4. Distribution of the rain gauges in Lazio and Umbria. These values are computed by running the (Basic) UDF rainGauges_density().

\subsection{Data Cleaning}

Using the (Basic) UDFs clean peaks() and clean_gauge () (to be invoked as: SELECT clean_peaks(3); SELECT clean_gauge $(3,30))$ it is

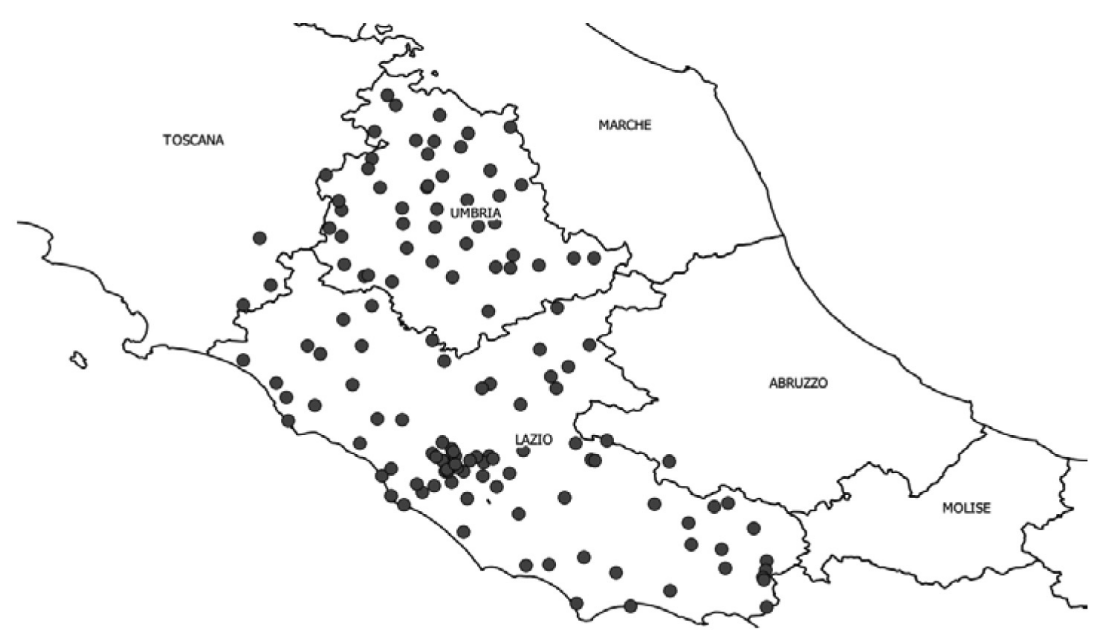

Figure 3. QGIS displaying the contents of the file of the rain gauges. 
possible to eliminate the outliers and the suspicious rain gauges as well. Specifically, the first query eliminates from table precipitation all the tuples that in the field accumulated have a value of the rainfall in 15 minutes greater than $3 \mathrm{~mm}$, while the second query eliminates from table rain_gauge the corresponding stations if it happens that they have a percentage of erroneous readings above $30 \%$. The arbitrarily set threshold value of $3 \mathrm{~mm}$ in 15 minutes of precipitation can be adjusted to the geographic region of reference.

\subsection{Measurements Plotting}

Diagrammatic visualization of the measurements collected in the SDB is a relevant need. In this section we show examples of what can be done very easily by invoking the UDFs being part of the package Plotting.

The query:

$$
\begin{gathered}
\text { SELECT plot_withinHours } \\
\text { ('plot_withinHours.pdf', 'Eur', } \\
\text { '2007-11-01', '00:00', '22:15') }
\end{gathered}
$$

returns, as a pdf file, the diagram of the rainfall measured by the rain gauge called 'Eur', located in the Trastevere area of Rome, from 00:00 to 22:15 on November $1^{\text {st }}, 2007$ (Figure 4).

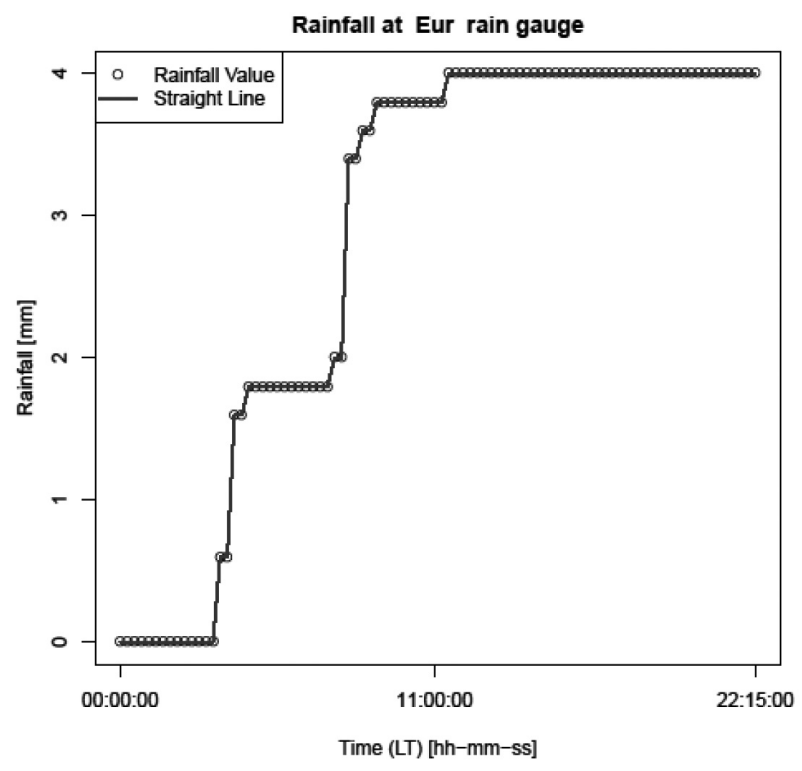

Figure 4. The rainfall measured by the rain gauge Eur on 2007-11-01, between 00:00 and 22:15.
The UDF plot_withinHours () (Appendix) uses UDF_1 and UDF_7 (Appendix). By calling the UDFs: plot_withinDays(), plot_withinMonths (), and plot_withinYears () it is possible to change the granularity of the diagram.

The barplot in Figure 5 shows the rainfall registered at each rain gauge in the Lazio region on 2007-11-01, from 00:00 to 23:00. The diagram is built by running the command:

SELECT barPlot_withinHours ('2007-11-01', '00:00', '23:00',

'barPlot_withinHours.pdf')

Each bar of the graph represents the precipitation accumulated in a rain gauge in the specified twenty-three hours.

To deepen the knowledge about the data behind the diagram of Figure 5, it would be sufficient to make recourse to the querying. Unfortunately, it is not reasonable to assume that nontechnical users have such a skill. But, we can help them by creating the view rgwithinlazio that hides in its "body" most details (namely, the need to look at three of the four database tables and join two of them, as well as the need to call the spatial operator ST_Contains() and the function SUM).

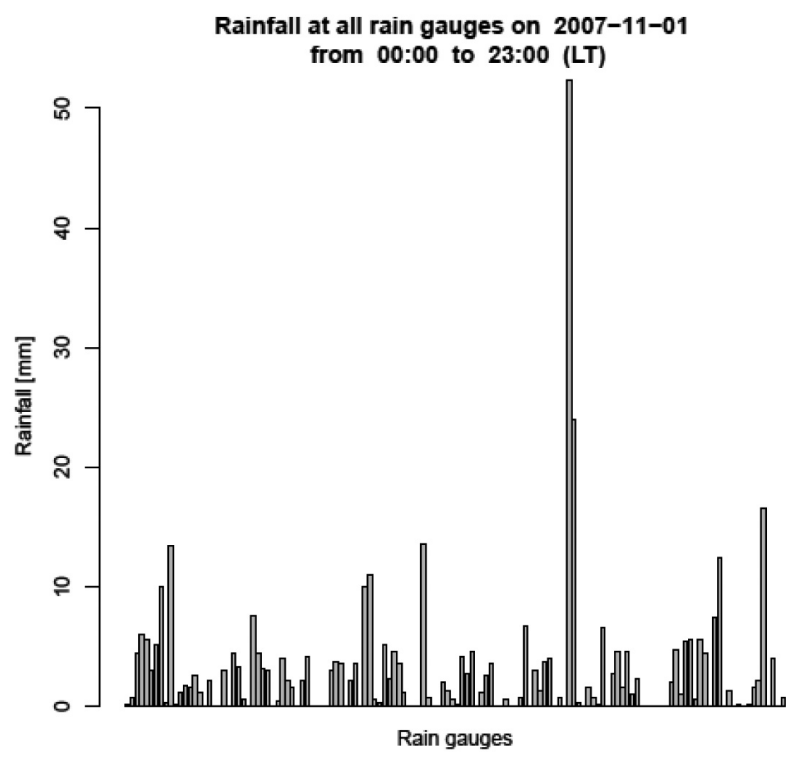

Figure 5. Time series of the precipitation measured by the rain gauges within Lazio on the $1^{\text {st }}$ of November 2007, from 00:00 to 23:00. (A similar histogram may be found in Haberlandt, 2007.) 
CREATE VIEW rgwithinlazio AS (

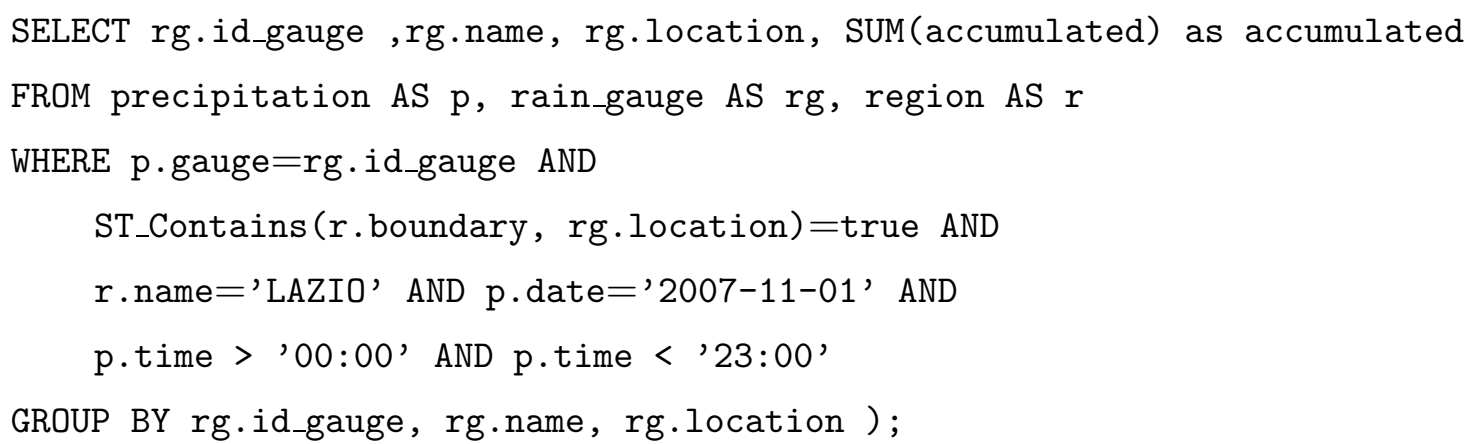

After a short on-the-job training, nontechnical users, by calling such a database object, may become able to formulate non-trivial queries such as, for instance, to count the stations that have measured a rainfall above a given threshold (e.g., $13 \mathrm{~mm}$ ) on 2007-11-01:

\section{SELECT count (id_gauge)}

FROM rgwithinlazio

WHERE accumulated > 13;

or display the identifier, name and geographic position of those stations:

\section{SELECT id_gauge name, location}

FROM rgwithinlazio

WHERE accumulated > 13;

The output of this second query can be displayed in the usual tabular format (Figure 6) as well as a map (Figure 7).

\begin{tabular}{|c|c|c|c|c|c|c|c|}
\hline \multicolumn{2}{|c|}{ Previous queries } & & & $v$ & Cancella & \multicolumn{2}{|c|}{ Cancella tutto } \\
\hline \multicolumn{2}{|c|}{$\begin{array}{l}\text { SELECT } \\
\text { FROM } \\
\text { WHERE }\end{array}$} & $\begin{array}{l}\text { gauge, } \\
\text { ithini } \\
\text { umulat }\end{array}$ & $\begin{array}{l}\text { ame, locatio } \\
10 \\
i>13\end{array}$ & & & & \\
\hline \multicolumn{2}{|l|}{$c$} & & & & & & $>$ \\
\hline \multicolumn{6}{|c|}{ Riquadro di soutputs } & & $\mathrm{x}$ \\
\hline \multicolumn{2}{|c|}{ Output dei dati } & Explain & Messaggi & Cronol & logia & & $\nabla$ \\
\hline & $\begin{array}{l}\text { id_gauge } \\
\text { integer }\end{array}$ & $\begin{array}{l}\text { name } \\
\text { charac }\end{array}$ & r varying $(50)$ & \multicolumn{3}{|c|}{$\begin{array}{l}\text { location } \\
\text { geometry }\end{array}$} & \\
\hline 1 & 13168 & Aureli & & \multicolumn{3}{|c|}{$0101000020 \mathrm{E} 61000009 \mathrm{~s}$} & \\
\hline 2 & 15228 & Vellet & & \multicolumn{3}{|c|}{$0101000020 \mathrm{E} 6100000 \mathrm{AS}$} & \\
\hline 3 & 18356 & Atina & & \multicolumn{3}{|c|}{$0101000020 \mathrm{E} 6100000 \mathrm{CF}$} & \\
\hline 4 & 18365 & Collef & rro & \multicolumn{3}{|c|}{$0101000020 \mathrm{E} 61000007 \mathrm{~s}$} & \\
\hline
\end{tabular}

Figure 6. The table collecting the identifier, name and geographic position of the rain gauges within Lazio that measured, on the $1^{\text {st }}$ of November 2007, a precipitation volume above $13 \mathrm{~mm}$.

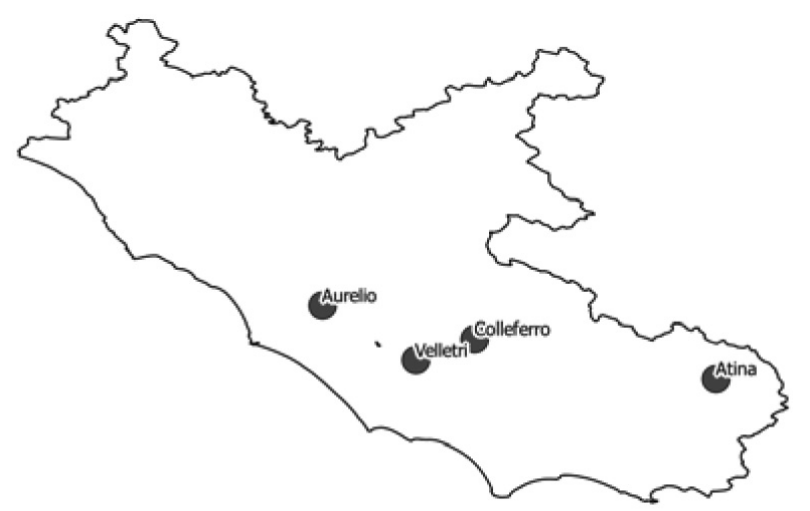

Figure 7. The (QGIS) map about the rain gauges within Lazio that measured, on the $1^{\text {st }}$ of November 2007, a precipitation volume above $13 \mathrm{~mm}$.

The UDFs rainfallPlot_withinHours(), rainfallPlot_withinDays(), rainfallPlot withinMonths (), rainfallPlot_withinYears() build diagrams that bring the progressive sum of the precipitation recorded in the time interval specified, as well as that fallen every 15 minutes.

\subsection{Interpolation}

In the reality, one always has to estimate the value of environmental variables from a limited set of measurements. For this reason many spatial interpolation methods have been developed. IDW and kriging are the best known methods (Li and Heap, 2008). As pointed out by Bostan et al. (2012), spatial interpolation methods have been applied to precipitation measurements on daily, monthly and annual averages. The IDW, Kriging, and Cross Validation (Table 3) packages support all those granularities. Below, we focus on the IDW method. 


\subsubsection{IDW}

This method is based on the physical law that everything is related to everything else, but near things are more related than distant things. IDW is one of the simplest and most popular interpolation techniques (Li and Heap, 2008).

The IDW method is very effective when applied to precipitation data, under the condition that the optimal exponent $(p)$ is identified $(\mathrm{Lu}$ and Wong, 2008), (Li et al., 2011), (Keblouti et al., 2012), (Chen and Liu, 2012). It is possible to determine the optimal value of $p$ minimizing the Root Mean Squared Error (RMSE).

The IDW package allows to interpolate the measured data (and collected in the table precipitation), adopting the four levels of granularity mentioned in Section 4. For example, via the UDF idw_whithinDays() (Appendix), it is possible to apply the IDW method to the values of the precipitation accumulated in an arbitrary number of days. The CrossValidation package supports the calculation of the RMSE through four routines referring to the four different temporal granularities supported by the packages of Table 3.

Through the UDF idw_crossValidation_withinDays() (Appendix), we calculated the RMSE for values of $p$ ranging between 0 and 10 , for six distinct daily intervals: $01-05,06-10,11-$ 15, 16-20, 21-25 and 26-30 of November 2007. The numerical results are collected in Table 5. It shows that values of $p$ between 1 and 3 return a very low error for each of the six time intervals

\begin{tabular}{|c||c|c|c|c|c|c|}
\cline { 2 - 7 } \multicolumn{1}{c|}{} & \multicolumn{6}{c|}{ RMSE } \\
\hline$p$ & $01-05$ & $06-10$ & $11-15$ & $16-20$ & $21-25$ & $26-30$ \\
\hline \hline 0 & 5.594 & 2.474 & 7.475 & 4.492 & 6.878 & 7.645 \\
\hline 1 & $\mathbf{5 . 3 9 8}$ & 2.212 & 7.060 & 3.449 & $\mathbf{6 . 7 6 6}$ & 5.069 \\
\hline 2 & 5.404 & 1.895 & 6.572 & $\mathbf{2 . 9 9 7}$ & $\mathbf{6 . 7 7 5}$ & 3.410 \\
\hline 3 & 5.423 & $\mathbf{1 . 8 0 3}$ & $\mathbf{6 . 4 5 8}$ & 3.068 & 6.926 & $\mathbf{3 . 2 4 0}$ \\
\hline 4 & 5.446 & 1.856 & 6.509 & 3.230 & 7.158 & 3.405 \\
\hline 5 & 5.472 & 1.919 & 6.578 & 3.345 & 7.393 & 3.549 \\
\hline 6 & 5.496 & 1.969 & 6.637 & 3.417 & 7.591 & 3.639 \\
\hline 7 & 5.516 & 2.006 & 6.682 & 3.465 & 7.746 & 3.690 \\
\hline 8 & 5.531 & 2.034 & 6.717 & 3.498 & 7.868 & 3.720 \\
\hline 9 & 5.544 & 2.055 & 6.742 & 3.522 & 7.966 & 3.739 \\
\hline 10 & 5.555 & 2.071 & 6.760 & 3.539 & 8.048 & 3.751 \\
\hline
\end{tabular}

Table 5. The RMSE values for different $p$ values. considered. This result is in line with the findings of studies already conducted. For instance, Chen and Liu (2012) found that the optimal exponent value varies between 0 and 5; Ahani et al. (2012) report a case study on precipitation in Iran for a period of 33 years for which they found that the optimum value of $p$ is 2 .

Set $p=2, n x=n y=300$ (to know about $n x$ and $n y$ please refer to the Appendix), we got (by using idw_whithinDays ()) the maps of the rainfall over Lazio, for the entire month of November 2007 (Figure 8).

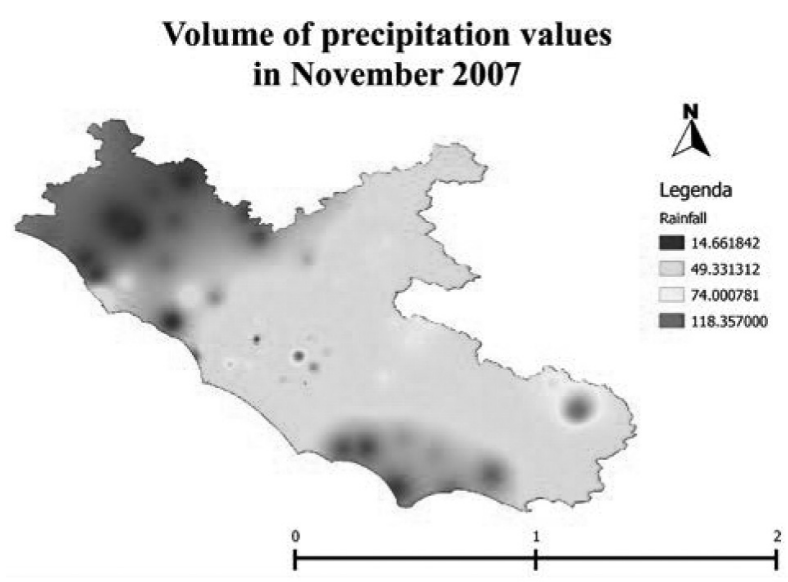

Figure 8. The raster map of the total precipitation volume over Lazio, in November 2007.

Before closing the section, we propose a comparison between the raster map in Figure 8, with that obtained by using QGIS (Figure 9). As can be seen, the two maps are almost identical.

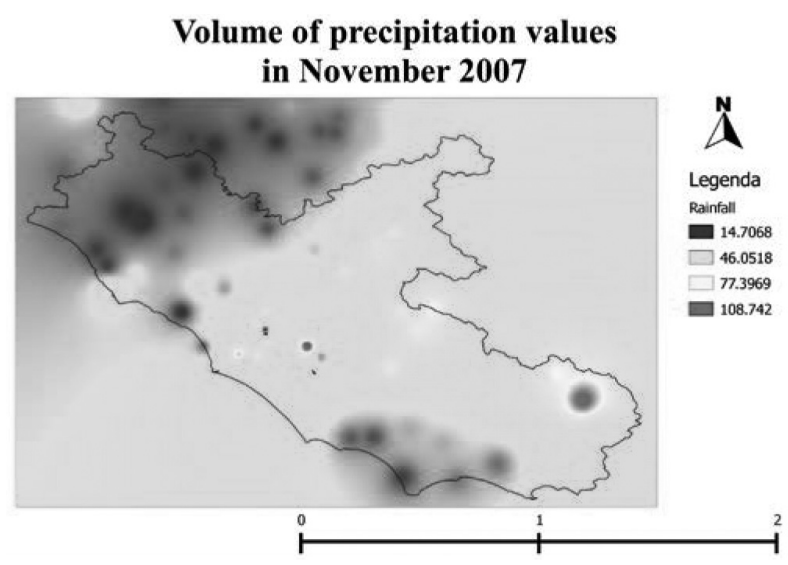

Figure 9. Result of the interpolation via QGIS. Notice that QGIS interpolates over a square region. 


\section{Conclusions}

The adopted solution setting aims at encouraging nontechnical users, after a short on-thejob training, to manipulate by themselves the measurements coming from rain gauges located over a geographical area. The involved stakeholders are only required to familiarize with the objects that the SDB exposes and invoke their execution.

Our vision for the near future hopes that national agencies with the responsibility of land government (in the case of Italy the Department of Civil Protection) will make available periodically (e.g. on an annual basis) an SDB about the historical rainfall data, enhanced with a wide repertoire of objects (views and UDFs) oriented to analyse those data. In addition to the SDB, it will be sufficient to provide a user's guide so that anyone who has the desire or the need can take advantage of this service to perform studies focused on the portions of the territory of his interest.

Last but not least, it is worthwhile to point out that the solution framework we have implemented to carry out the experience reported in the paper can be replicated in other areas of high social impact that involve large spatio-temporal data, keeping the operational effectiveness unaltered.

\section{References}

[1] H. Ahani et AL., An investigation of trends in precipitation volume for the last three decades in different regions of Fars province, Iran. Theoretical and Applied Climatology, 109 (2012), 361-382.

[2] P. A. Bostan, G. B. M. Heuvelink, S. Z. AKYUREK, Comparison of regression and kriging techniques for mapping the average annual precipitation in Turkey. International Journal of Applied Earth Observation and Geoinformation, 19 (2012), 115-126.

[3] D. J. Burnette, D. W. Stahle, Computer assisted screening, correction, and analysis of historical weather measurements. Computers \& Geosciences, 54 (2013), 309-317.

[4] A. J. CANNON, Quantile regression neural networks: Implementation in $\mathrm{R}$ and application to precipitation downscaling, Computers \& Geosciences, 37 (2011), 1277-1284.
[5] C. J. Carleton, R. A. Dahlgren, K. W. Tate, A relational database for the monitoring and analysis of watershed hydrologic functions: I. Database design and pertinent queries. Computers \& Geosciences, 31 (2005), 393-402.

[6] F. W. CHEN, C.-W. LIU, Estimation of the spatial rainfall distribution using inverse distance weighting (IDW) in the middle of Taiwan. Paddy and Water Environment, 10 (2012), 209-222.

[7] U. HABERLANDT, Geostatistical interpolation of hourly precipitation from rain gauges and radar for a large-scale extreme rainfall event. Journal of Hydrology, 332 (2007), 144-157.

[8] T. Hengl, A Practical Guide to Geostatistical Mapping. Office for Official Publications of the European Communities, Luxembourg, 2009.

[9] M. Keblouti, L. Ouerdachi, H. Boutaghane, Spatial Interpolation of Annual Precipitation in Annaba-Algeria - Comparison and Evaluation of Methods. Energy Procedia, 18 (2012), 468-475.

[10] J. LI, A. D. HEAP, A Review of Spatial Interpolation Methods for Environmental Scientists. Geo-science Australia Record, 23 (2008), 137.

[11] Q. LI, Y. CHEN, Y. SHEN, X. LI, J. XU, Spatial and temporal trends of climate change in Xinjiang, China. Journal of Geographical Science, 21(6) (2011), 1007-1018.

[12] G. Y. Lu, D. W. Wong, An adaptive inverse-distance weighting spatial interpolation technique. Computers \& Geosciences, 34 (2008), 1044-1055.

[13] E. J. Pebesma, Multivariable geostatistics in S: the gstat package. Computers \& Geosciences, 30 (2004), 683-691.

[14] J. POKORNY, Database architectures: Current trends and their relationships to environmental data management. Environmental Modelling \& Software, 21 (2006), 1579-1586.

[15] P. Rowhania, D. B. LOBELL, M. LindERMANC, N. RAMANKUTTYA, Climate variability and crop production in Tanzania. Agricultural and Forest Meteorology, 151 (2011), 449-460.

[16] J. SHEN, A. PARKer, J. Riverson, A new approach for a Windows-based watershed modeling system based on a database-supporting architecture. Environmental Modelling \& Software, 20 (2005), 1127-1138.

[17] S. Steiniger, E. Bocher, An Overview on Current Free and 61 Open Source Desktop GIS Developments. International Journal of Geographical Information Science, 23 (2009), 1345-1370.

[18] H. TABari, P. H. Talaee, Temporal variability of precipitation over Iran: 1966-2005. Journal of Hydrology, 396 (2011), 313-320.

[19] M. O. Ward, W. L. Power, P. KetelaAr, A computational environment for the management, processing, and analysis of geological data. Computers \& Geosciences, (1996), 1123-1131. 
Received: January, 2014

Revised: May, 2014

Accepted: June, 2014

Contact addresses:

Paolino Di Felice

Department of Ingegneria Industriale e dell'Informazione ed Economia

University of L'Aquila

Italy

e-mail: paolino.difelice@univaq.it

Luca Finocchio

Daniele Leombruni

Vittoriano Muttillo

Master students in Engineering Informatics

University of L'Aquila

Italy
PAOLINO DI FELICE is a professor of computer science at the Department of Industrial and Information Engineering \& Economics of the University of L'Aquila, since 1999. He has authored or co-authored about 100 articles in international journals, books, and conference proceedings in the areas of programming methodologies, relational and spatial databases. He has also carried out a consistent activity of technological transfer in collaboration with several IT firms. His research has been funded by national and international institutions and carried out in collaboration with researchers from several countries (Italy, Holland, Germany, and the USA). He has served within national and international committees. For the last few years, he has been a regular member of the program committee of the International Conference on Integrated Information (IC-ININFO).

LUCA FinOCCHIO, DANIELE LEOMBRUNI AND VitTORIANO MUTTILlO are Master students of engineering informatics at the University of L'Aquila (Italy). 


\section{Appendix}

The SQL/DDL statements to create the four tables of the SDB (Section 3).

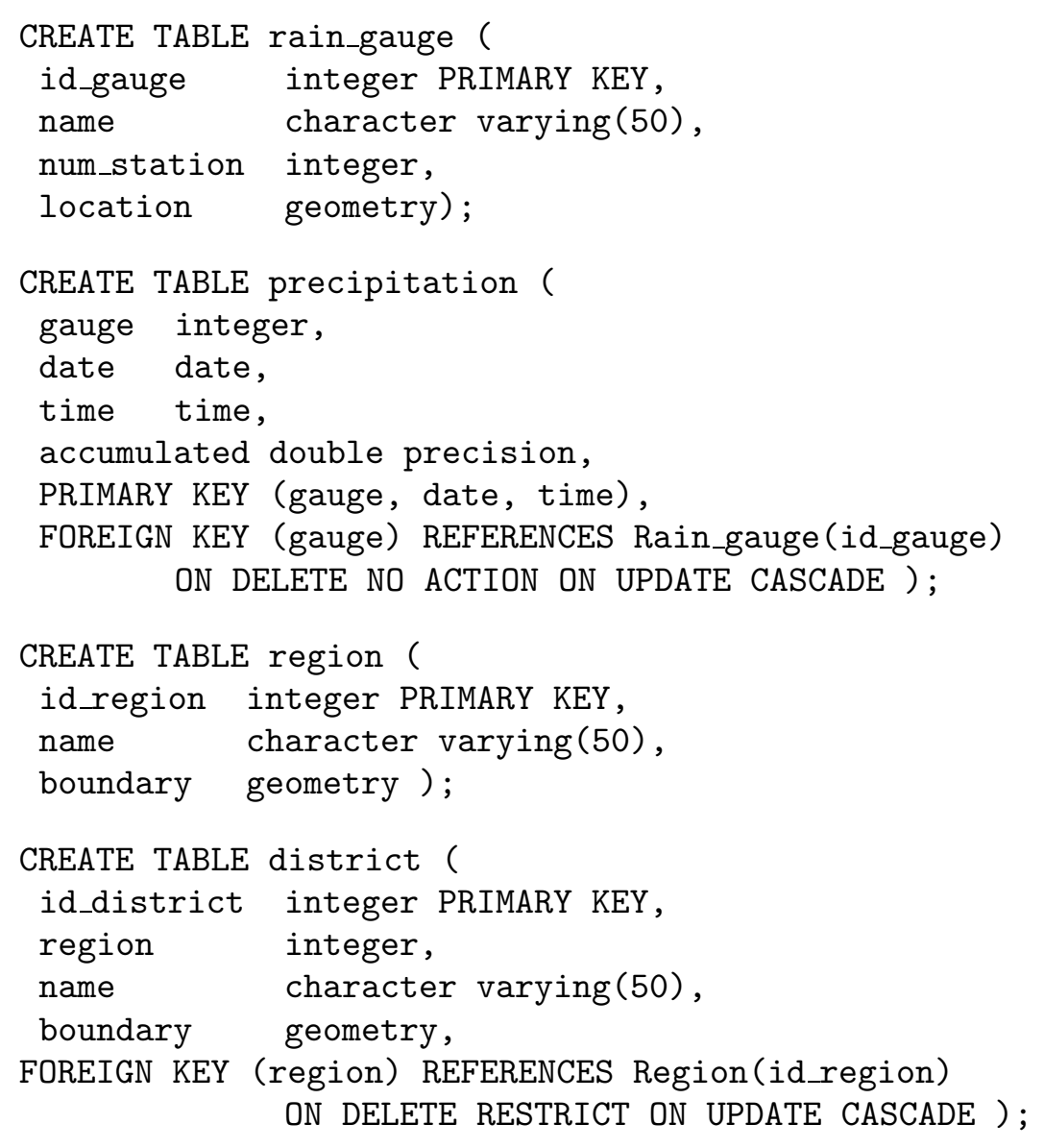

The remainder of this appendix collects the code of some of the UDFs mentioned in Section 5.

The UDF import_precipitation().

It migrates the data about the rain measurements into table precipitation.

Inputs:

- $\operatorname{path}($ text): path to reach the file of the measurements data

- date (text): month to which the measurements refer (date format: yyyy-mm).

CREATE OR REPLACE FUNCTION import_precipitation(path text, date text)

RETURNS void AS

\$BODY\$

INSERT INTO precipitation (gauge, date, time, accumulated)

SELECT gauge, date, time, accumulated

FROM UDF_O (path, date)

$t$ (gauge integer, time time, station text,

\$BODY\$ accumulated double precision, date date)

LANGUAGE SQL

The (PL/R) UDF_O returns a set of records, each composed of five values, without specifying for them a strucure in the usual relational sense. The command $t(\ldots)$ allows to assign to each value within the generic record a "header", specifying for each column the name and the data type. 


\section{$U D F_{-} 1$}

It calculates the sums of the progressive accumulated rain every 15 minutes in a given rain gauge in a predetermined interval of hours of a specific day.

\section{Inputs:}

- gauge_name (text): name of the rain gauge

- day (text): the day of interest

- from_h (text): the initial hour

- to h (text): the final hour

\section{Output:}

A set of tuples of type rainfall.

rainfall is a user data type defined as follows:

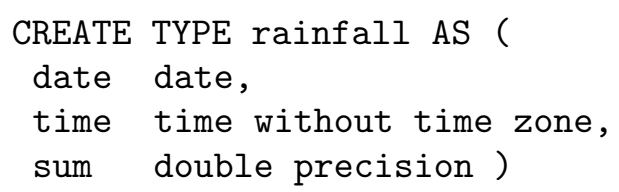

\section{The PL/pgSQL code:}

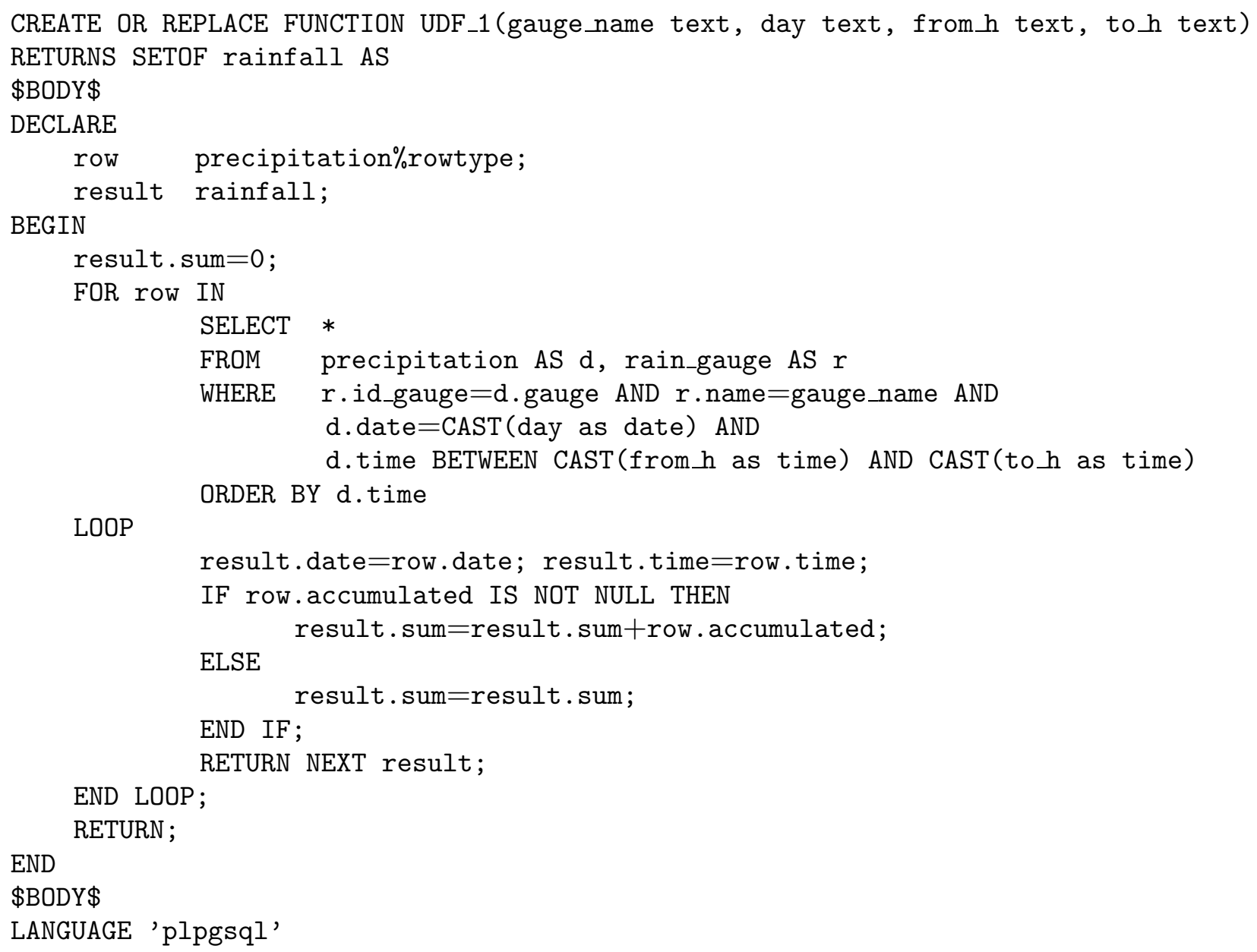




\section{$U D F_{-} 7$}

It returns a .pdf file containing the chart with the hours on the $x$-axis and the amount of rainfall measured $(\mathrm{mm})$ on the $y$-axis.

\section{Inputs:}

- path (text): path of the (.pdf) file to be created

- gauge_name (text): name of the pluviometer of interest

- $\mathrm{x} 1$ (time []): array about the time of sampling

- y (double precision[]): array of the measurements

\section{Output:}

A boolean value: true if the pdf file has been created, false otherwise

\section{The PL/R code:}

CREATE OR REPLACE FUNCTION UDF_7 (path text, gauge_name text, date text,

\section{RETURNS boolean AS}

$$
\mathrm{x} 1 \text { time without time zone[], y double precision[]) }
$$

\section{\$BODY\$}

$\mathrm{x} 2<-\operatorname{strptime}\left(\mathrm{x} 1\right.$, format= $\left.\% \mathrm{H}: \% \mathrm{M}^{\prime}\right)$;

$\mathrm{x}<-$ as.double $(\mathrm{x} 2)$;

$1<-\operatorname{length}(x)$;

$\operatorname{pdf}($ path);

plot (x, y, xlab='Time (LT) [hh-mm-ss]', ylab='Rainfall [mm]',

main=paste('Rainfall at', gauge name, ' rain gauge on ', date), xaxt='n');

$\operatorname{axis}(1, \operatorname{at}=c(x[1], x[1 / 2], x[1])$, labels=c $(x 1[1], x 1[1 / 2], x 1[1]))$;

lines (x, y, col='blue', lwd=2);

legend ( $x[1], \max (y), c($ Rainfall Value', ' Zigzag Curve'), Ity=c $(-1,1), \operatorname{pch}=c(1,-1)$, col=c ('black', 'blue'), lwd=c $(-1,2)$, merge=TRUE);

$\operatorname{dev}$.off ();

return (TRUE);

\$BODY\$

LANGUAGE 'plr';

\section{The UDF plot_withinHours()}

It plots the sum of the rainfall accumulated every 15 minutes in a rain gauge in a range of hours of a given day. The drawing is returned as a .pdf file.

Inputs:

- path (text): path of the (.pdf) file to be created

- gauge_name (text): name of the rain gauge

- from_h (text): the initial hour

- to $\mathrm{h}$ (text): the final hour

\section{Output:}

A boolean value: true if the pdf file has been created, false otherwise 
The PL/pgSQL code:

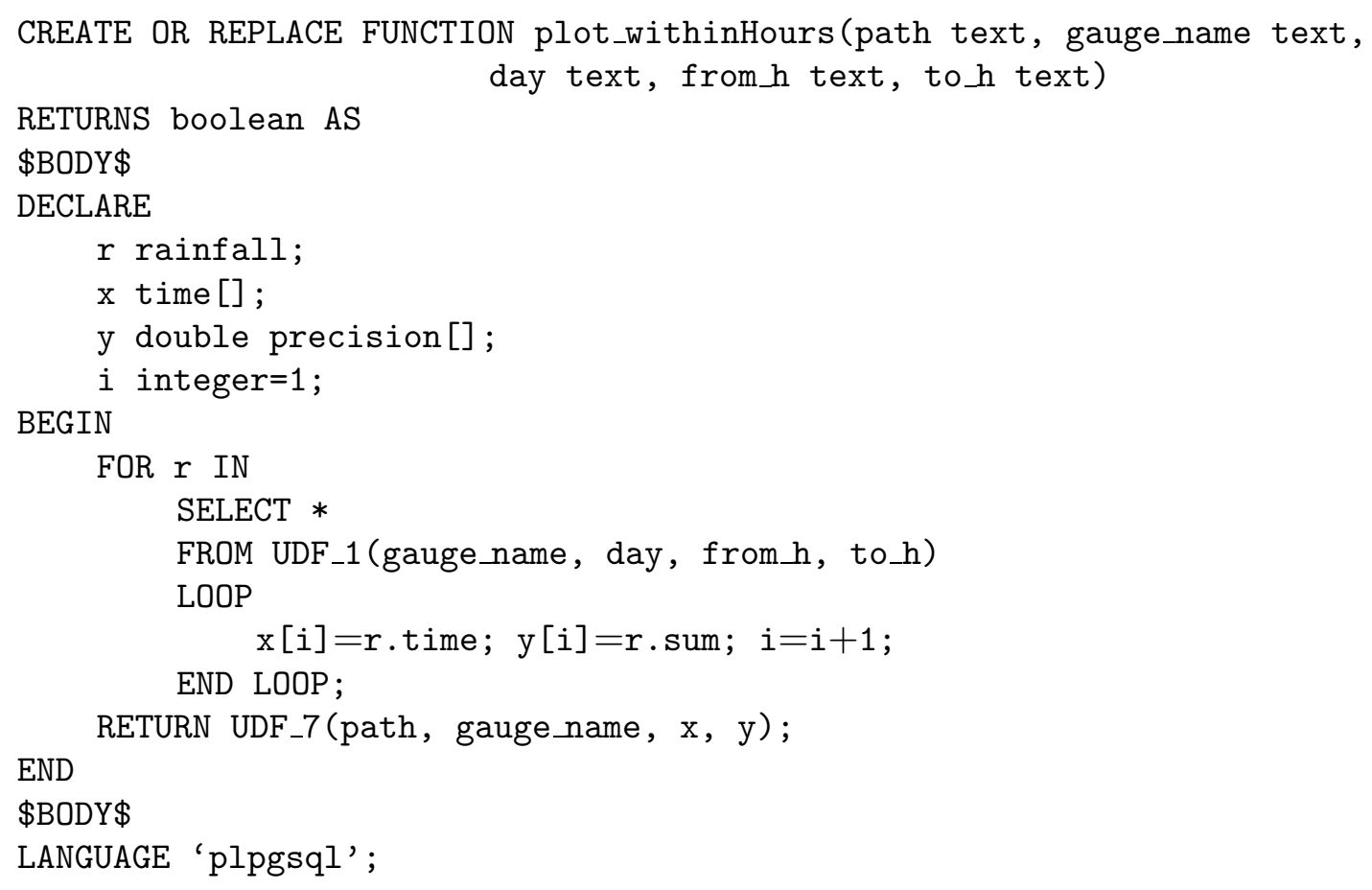

\section{The UDF idw_withinDays ()}

It interpolates, by means of the IDW technique, the rain accumulated in all the rain gauges in a given interval of days within a given region (in our case, either Lazio or Umbria). A geocoded (.asc) raster file is returned. This function calls four (Auxiliary) UDFs. The (PLpgSQL) UDF_4 computes the sum of the precipitation accumulated by all the rain gauges in the given interval of days within the given region. The (PL/R) UDF_14 computes the interpolation grid, given the dimensions and the coordinates $x$ and $y$ of a square. The (PLpgSQL) UDF_15 filters the points of the grid returned by UDF_14 within the given region. The (PL/R) UDF_16 interpolates, by calling the function idw of the library gstat, the data received as inputs and returns a geocoded (.asc) raster file built by linking the function raster of the library raster.

\section{Inputs:}

- region_name (text):

name of the region of interest (either Lazio or Umbria)

- from_d (text): the initial day

- to_d (text): the final day

- path (text): path of the (.pdf) file to be created

- power (double precision): value of the exponent of the IDW method

- $\mathrm{nx}$ (int): number of points of the grid along the $x$-axis

- ny (int): number of points of the grid along the $y$-axis

\section{Output:}

The actual number of points interpolated. 
The PL/pgSQL code:

CREATE OR REPLACE FUNCTION idw_whithinDays(region_name text, from_d text, to_d text, RETURNS integer AS path text, power double precision, $\mathrm{nx}$ integer, ny integer)

\$BODY\$

DECLARE

id_gau integer [];

gauge_na character varying(50) [];

acc double precision [];

row record;

$\mathrm{x}$ double precision [];

y double precision [];

$i$ integer;

row1 record;

$\mathrm{xg}$ double precision[];

yg double precision [];

ig integer;

row2 record;

xgf double precision[];

ygf double precision[];

BEGIN

igf integer;

$i=0$;

FOR rOw IN

SELECT id_gauge AS id, gauge_name AS name, sum AS a, ST X(location) AS xx, ST_Y(location) AS yy

LOOP

FROM UDF_4 (from_d, to_d)

id_gau [i] =row.id;

gauge_na $[i]=$ row . name;

$x[i]=$ row $\cdot x x$;

$y[i]=$ row $\cdot y y$;

acc $[i]=$ row . a;

$i=i+1$;

END LOOP;

ig $=0$;

FOR rOw1 IN

SELECT *

FROM UDF_14(11, 14.5, 40.5, 44, nx, ny)

LOOP t(xgrid double precision, ygrid double precision)

$\mathrm{xg}[\mathrm{ig}]=$ row1.xgrid;

yg $[$ ig $]=$ row1 ygrid;

$i g=i g+1$;

END LOOP;

igf $=0$;

FOR row2 IN

SELECT

FROM UDF_15 (region_name, $x g, y g$ )

$t$ (is_in boolean, xgridfilter double precision, ygridfilter double precision) 
LOOP

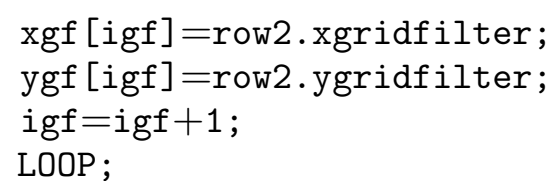

END;

RETURN UDF_16(id_gau, gauge_na, $x, y, x g f, y g f$, acc, power, path);

\$BODY\$

LANGUAGE plpgsql;

The UDF idw_crossValidation_withinDays()

It performs the cross validation of the IDW method, run by changing the value of the exponent. All the interpolations refer to the same interval of days given as input. The cross validation is coded inside the (PL/R) UDF_17, which calls specific gstat functions that carry out the actual computations.

\section{Inputs:}

- from_d (text): initial day

- to_d (text): final day

- fromp (double precision): lower bound of the exponent

- to_p (double precision): upper bound of the exponent

- by_p (double precision): step of increment of the exponent

\section{Output:}

A set of records about the RMSE for each exponent value.

The PL/pgSQL code:

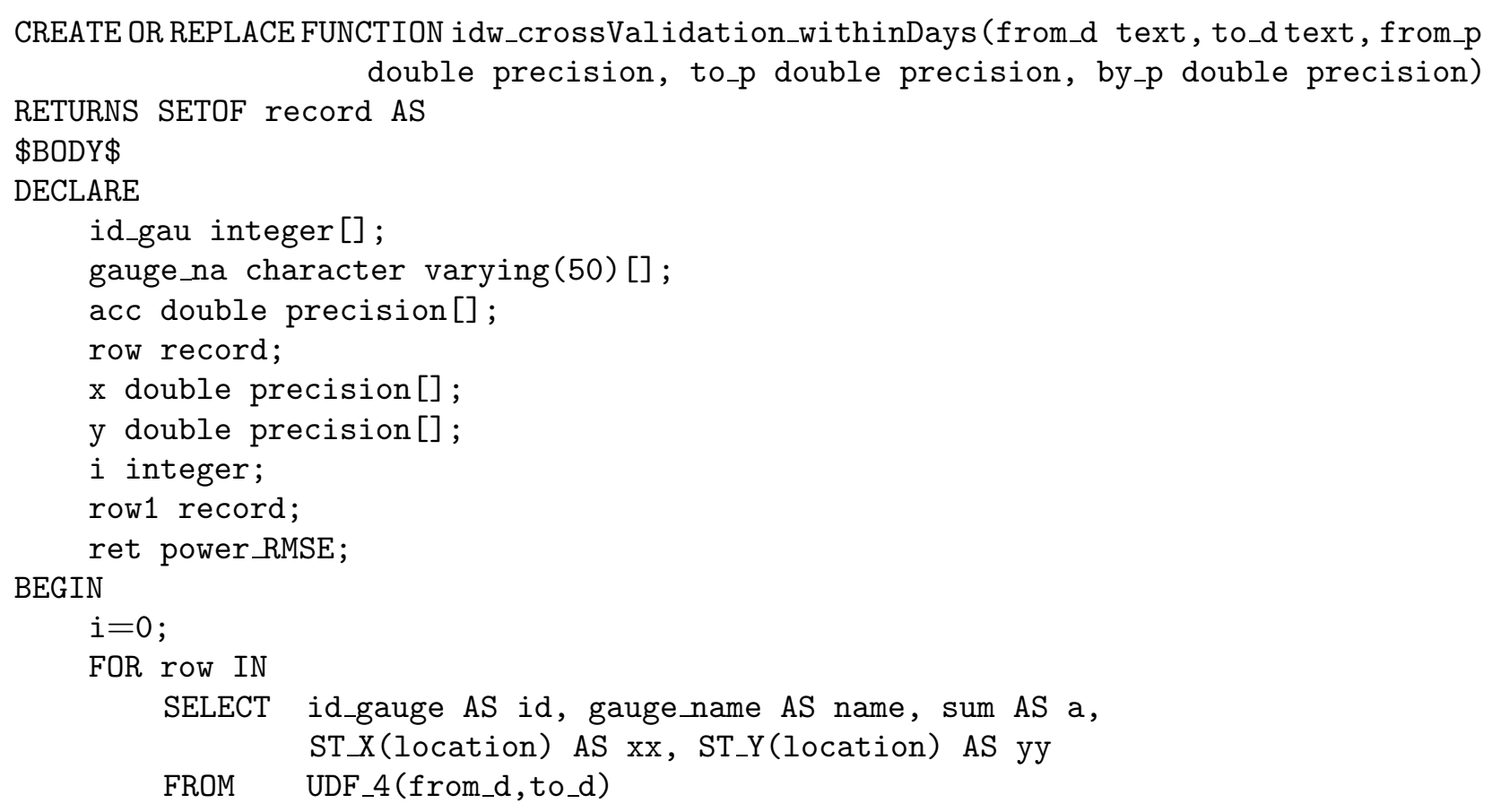




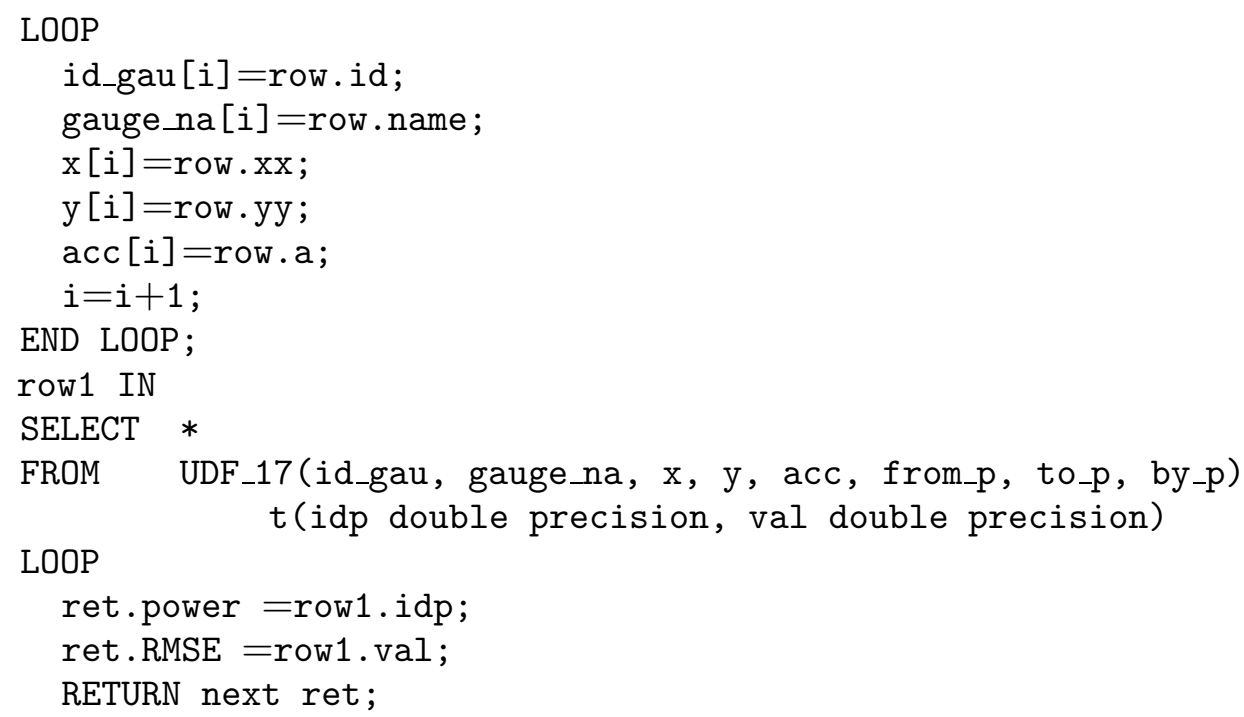

